\title{
Performance Comparison of Cloud Computing/IoT Virtualization Software, Hyper-V vs vSphere
}

\author{
Samad Salehi Kolahi, Vichar S. Hora, Amrit P. Singh, Sherazia Bhatti, Susmitha R. Yeeda \\ Unitec Institute of Technology, Auckland, New Zealand \\ skolahi@unitec.ac.nz
}

\begin{abstract}
Hyper-V and vSphere are amongst the top virtualization software's available in the market currently. Both of these products are from different vendors, Hyper-V is developed by Microsoft and vSphere is devolved by VMware. The intent of this paper is to compare the network performance of two different software's using IPv4 and IPv6 that will allow us to determine which perform better at different stages of the test. Virtualization is used in Cloud data centers that store most of IoT (Internet of Things) data. Results show VMware (vSphere) is able to achieve much higher throughput that Hyper-V. Also VMWare showed lower delay, but the differences is in milliseconds that is not noticeable.
\end{abstract}

Keywords: Virtualization, IoT, Cloud Computing, VMware (vSphere), Hyper-V

\section{INTRODUCTION}

One of the modern/future technologies is expected to be is Internet of Things (IoT) where devices, computers, mobiles, are networked with users. Among the technologies used in the IoT is Cloud Computing that keeps the IoT servers in data centers. These servers will be virtualized servers. Virtualization technology creates a virtual IT environment without the need of any additional physical hardware. Vitalization in computing has been introduced to the computing world some time ago, but we are able to fully utilize the capabilities of virtualization now because we do not lack hardware and software. It's a fact that computer hardware/software that was expensive many years ago, it now only worth much less. Virtualization was expensive when it was introduced only certain persons were able to afford to fund such a technology. But today, it is even possible for small businesses to run virtualization software.

Vitalization is being used a lot in the IoT and cloud computing [1] because of better efficiency. Less capital, less energy utilization, less physically equipment's being used provides the benefit of less energy consumption. Less physical space required to maintain the network [2]. Virtualization has many benefits when cost is a high priority. In many cases, what many large organizations are looking for is low cost high productivity. Different virtualization software's have different performance results, some aiming their product high in performance others, in user-friendly. In this paper our focus is to conduct network performance tests for visualization software (Hyper-V and vSphere) using TCP, UDP protocols, IPv4 and IPv6 protocols. Our findings will state which virtualization software provides the best throughput using various protocols. Hyper-V [3] vs vSphere [4] is a highlighted topic of virtualization. Both hypervisors are created by different manufacturers along with different architectural differences between the two. Hyper- $\mathrm{V}$ is based on a micro-kernalized architecture whereas VMware vSphere is based on monolithic architecture.

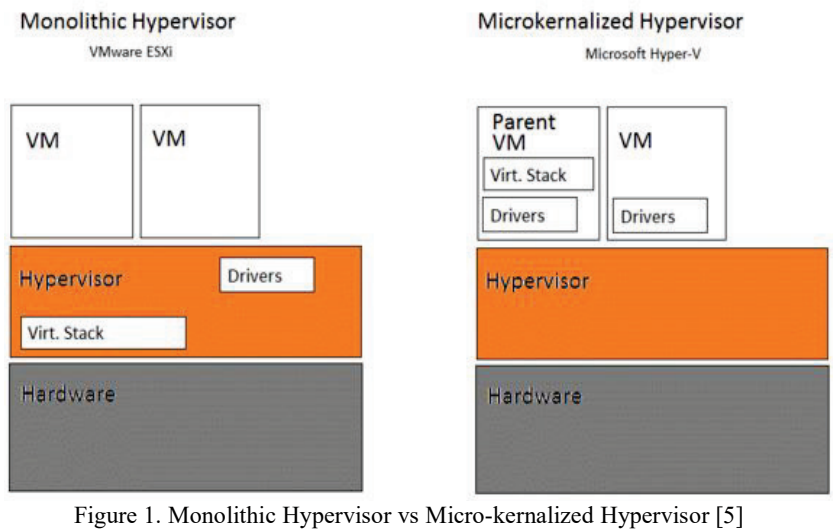

In Figure 1, it shows the architecture for both hypervisors. Monolithic hypervisors have the drivers and virtual stacks placed within the hypervisors above the hardware layer. Whereas micro-kernalized hypervisors have the drivers and virtual stacks sitting above the hypervisor layer [5]. Due to the architectural difference between the two hypervisors, it is possible for the hypervisors to provide with different results in various performances conducted [6].

In this study, we will measure the throughput, Round Trip delay Time (RTT) and Central Processing Unit Utilization (CPU Utilization) for IPv4 and IPv6 and virtualization software's mentioned.

The motivation behind this work is produce some new results in virtualization software evaluation, and IPv6, that are used in IoT/cloud environment. This paper helps organizations that are after better network performance from virtualization software's and vendors who have created the product for future developments.

The organization of this paper is as follows. This section introduced the topic and motivation for the work. 
In section II some of the related works is reviewed where the key focus is on performance. Section three is the network set up and monitoring tool used. In section IV the results that is produced from the tests are discussed. The section after that is conclusions, followed by future works, and references.

\section{RELATED WORK}

Research has been previously done via researchers on virtualization performance using various types of software's used in the market. Some of the related work are as follows.

In 2018 [7], the performance analysis of two hypervisor type-1 is compared, VMware ESXi and Microsoft Hyper-V. The authors concluded that for the small blocks and transfers workloads, Hyper-V and ESXi pefromance is very close. Although in some cases ESXi (full hardware emulation) shows better performances. The authors considering all possible tested cases and circumstances, recommend to choose Hyper-V rather than the ESXi. In 2016 [8], authors assessed the performance of four hypervisors: KVM, Hyper$\mathrm{V}$, ESXi and Xen response on SQL workload. They measure the performance with some traffic workload measuring parameters like delay Time, Processor CPU, RAM Memory and Storage. The performance results show that hypervisors do not have large performance variation with each other on various parameters. In 2014, AL Jabry et al. [9] Conducted a similar research on evaluation of the performance of virtualization technologies. They measured the performance of various aspects of the virtual machines such as, disk I/O, Memory and CPU utilization. They used tools such as Dhrystone, Whetstone and Excel Throughput. Throughput results for Dhrystone were shown the highest for three different virtualization software's such as VMware, Virtual Box and VirtualPC with the CPU utilization shown at $16 \%$ and above. The other tools have shown that CPU utilization is under $8 \%$ overall. In 2008, Walters et al. [10] Presented a similar research on the comparison of virtualization technologies for High performance Computers (HPC). Netperf was the tool used to do the performance testing. They used the TCP protocol to conduct the throughput and latency with various packet sizes (bytes). In latency it was shown as the packet size increased the latency measured in milliseconds went gradually higher from 256 Bytes packet size, the latency had significantly increased for all virtual software's. Throughput using TCP shows XEN with the highest throughput of $900 \mathrm{Mbit} / \mathrm{s}$, VMware and OpenVZ are below the $400 \mathrm{Mbit} / \mathrm{s}$ mark. A performance evaluation study was presented in 2008 by $\mathrm{Xu}$ et al. [11]. Their tests comparisons were carried out using benchmarks on the overall performance using Xen, KVM and VMware. They measured the efficiency of the virtualization technologies. They used a tool called Netperf as a server to do the performance; they found that the throughput performance of VMware and XEN virtualization software performance is equivalent to non-virtualized environment performance. VMware and XEN showed performance at $90+\mathrm{Mbit} / \mathrm{s}$ also corresponding to the physical machine which was also 90 Mbit/s.

\section{NETWORK SETUP}

The network test-bed was setup via switch port connected with standard category 5e cabling between three different machines. First machine being the server that has $\mathrm{AD}$, DNS and DHCP services installed. We installed vSphere client tool to access the VMs remotely via gigabit switch cable. PC1 we installed the vSphere with windows 10 as VM instance acting as the server. PC2 also has the vSphere installed with windows $10 \mathrm{VM}$ instance acting as client. Similar setup has been done in Hyper-V, only Hyper$\mathrm{V}$ management tool is used to access the VMs remotely.

The hardware specifications for both machines (server and client) are as follows: Desktop - Intel core i7 4790, 16 GB RAM, 3.60 GHz processor, 500 GB Hard Drive with a 64 bit Hyper-V bare metal. VMs - Intel core i7 4790, 4 GB RAM, 3.60 GHz processor (100\%), 50 GB Hard Drive with a 64-bit operating system and VMware vSphere. (Compatible NIC card installed). Cables was Straight through Category 5e and Switch was $1 \mathrm{Gbit} / \mathrm{s}$ switch with 8 ports.

The clients were all installed, using windows 10 professional operating system. The network performance was taken via a tool called Netperf [12]. Netperf was the ideal choice due to no compatibility issues. As windows 10 was recently released and Netperf was able to provide data without any issue. We have used 10 different packet sizes for the tests $(128,256,384,512,640,768,896,1024,1152$ and 1408 Bytes).

Each packet test was run for 3 minutes each run 20 times, resulting in 20 different figures each figure had a result of 10 figures averaged. This gave approximately results with $95 \%$ confidence interval. We wrote scripts for RTT, CPU Utilization and Throughput making it efficient to take results simultaneously. Results have been taken on desktop based machines with no additional settings or software's installed; only required software were used to determine the pure results.

\section{RESULT}

The results include the findings for our tests for throughput, RTT and CPU utilization of TCP and UDP protocols with IPv4 and IPv6 addressing protocols. All results recorded and documented for the comparison are explained additionally in the following graphs. 


\section{Throughput}

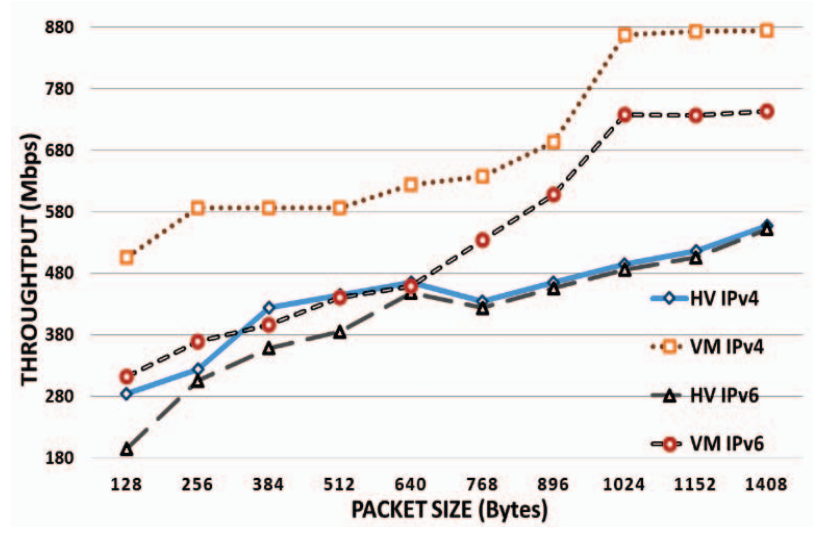

Figure 2: TCP Hyper-V (HV) vs VMware (VM)

In Figure 2, we can see the TCP throughput for Hyper-V and vSphere (VMWare) using both protocols, IPv4 and IPv6. For smaller packets ranging from 128 to 640 Bytes for Hyper-V TCP IPv4 we had a gradual increase in throughput ranging between 20-30 Mbit/s increase in each packet except between packet 256 and 384 Bytes with a difference of over $40 \mathrm{Mbit} / \mathrm{s}$. We found that on every packet ranging from 128 to 640 Bytes on VMware TCP IPv4 have a higher increase between each packet size. The average difference between the packets is ranging between 35-45 Mbit/s. Which in comparison to Hyper-V throughput VMware is producing much more on each packet.

TCP packets starting from 768 and 1408 Bytes for Hyper-V IPv4 are on an incline increase. Packet size 768 Bytes has dropped in throughput compared to the 640 Bytes packet size. In Figure 2, looking at VMware TCP IPv4 throughput increase from 768 to 1408 Bytes is significant getting higher from packet 1024 up to 1408 Bytes, the increase from packet 896 to 1024 is over $200 \mathrm{Mbit} / \mathrm{s}$. We found that Hyper-V IPv4 and VMware IPv4 results are very different. Evidently VMware is providing more throughput than Hyper-V in TCP IPv4 results. This is different results from [8] that found hypervisors behave the same.

We also noticed in our previous works [13, 14] that throughput and RTT graphs increase and decrease as packet size increases, and the graph is not smooth, but the general trend is increase in throughput with packet size as we note in Figures 2-5.

As seen in Figure 2 Hyper-V TCP IPv6 throughput results ranging from packet 128 to 640 Bytes is a very slow increase in throughput except from packet size 128 where the lowest throughput has been 194.5 Mbit/s whereas 256 Bytes packet size throughputs resulted in $305.7 \mathrm{Mbit} / \mathrm{s}$, showing over $100 \mathrm{Mbit} / \mathrm{s}$ increase in throughput. We found that the increase from the smaller packets (128 - 640 Bytes) is ranging from 15- $25 \mathrm{Mbit} / \mathrm{s}$. VMware TCP IPv6 results from packet size 128 to 640 Bytes are very stable from the beginning with only 30-40 Mbit/s including the lowest throughput on packet size 128 Bytes. Larger packets of Hyper-V TCP IPv6 from 768 to 1408 Bytes were on a steady incline of 35-45 Mbit/s on each packet. We found only packet 768 and 896 Bytes are showing a decline in throughput compared to packet size 640 Bytes as seen in Figure 2, while looking at the higher packet sizes we noticed that it's a steady incline. VMware TCP IPv6 results ranging from 768 to 1408 Bytes packet sizes showed incline in the throughput performance. The increase in each packet size is ranging from 35-45 Mbit/s. By looking at the overall comparison results between two different software's and vendors; VMware is resulting in much higher performance than Hyper-V.

We have found that the results of IPv4 and IPv6 are also very different when they were used for Hyper-V and VMware. IPv6 tents to show less performance in throughput than IPv4 on both hypervisors, due to higher overhead in IPv6. Our results show that VMware is performing better in providing higher throughput than Hyper-V in both TCP and UDP for both IPv4 and IPv6.

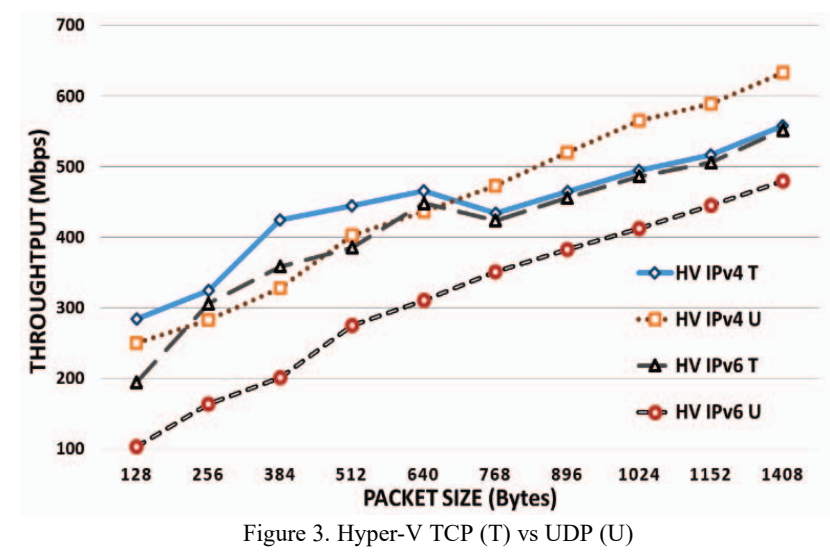

Figure 3 results are for Hyper-V TCP (T in diagram) and UDP results for IPv4 and IPv6. We found that packets ranging from 128 to 640 Bytes on Hyper-V TCP IPv4 have higher throughput results on each packet size with an average of 75-80 Mbit/s increase in throughput. Looking at Hyper-V UDP, IPv4 packet size 128 to 640 Bytes, the throughput results are gradually increasing. Though being lower than TCP at this stage. Packet size 768 to 1408 there has been a drop in throughput for Hyper-V TCP IPv4 results between packet 640 and 768 Bytes of $21 \mathrm{Mbit} / \mathrm{s}$, rest of the packets had a steady increase of throughput results. HyperV UDP, IPv4 results show a very steady increase unlike TCP of packet size 768 to 1408 Bytes averaging on between 55$60 \mathrm{Mbit} / \mathrm{s}$ increase in throughput with the highest throughput obtained at packet size 1408 of $636.7 \mathrm{Mbps}$ surpassing TCP IPv4 highest results with well over 60-70 Mbit/s increase. 
We found that TCP IPv4 results for Hyper-V are showing higher throughput in lower size packets where as UDP, IPv4 results are showing higher throughput in larger packet sizes ranging from 768-1408 Bytes. Results obtained for IPv6 TCP show higher increase in throughput from packet size 128 to 640 Bytes, the average increase between those smaller packet sizes has been about $80-90 \mathrm{Mbit} / \mathrm{s}$ increase in total. Hyper-V UDP IPv6 results show a very steady increase in through from the smaller packets averaging about 75-80 Mbit/s increase in total. On packet size 640 and 768 Bytes the throughput was on decline by $24 \mathrm{Mbit} / \mathrm{s}$ similar to IPv4 results in TCP. Larger packets have shown a substantial increase. IPv6 UDP results in the figure show a very stable increase even from larger packets, but much lower throughput results than TCP IPv6 largest packet on TCP IPv6 show $551.8 \mathrm{Mbit} / \mathrm{s}$ throughputs results whereas IPv6 UDP highest packet size result is only $479.4 \mathrm{Mbit} / \mathrm{s}$. From the results we found UDP, IPv4 is better than TCP and in IPV6 TCP is better than UDP.

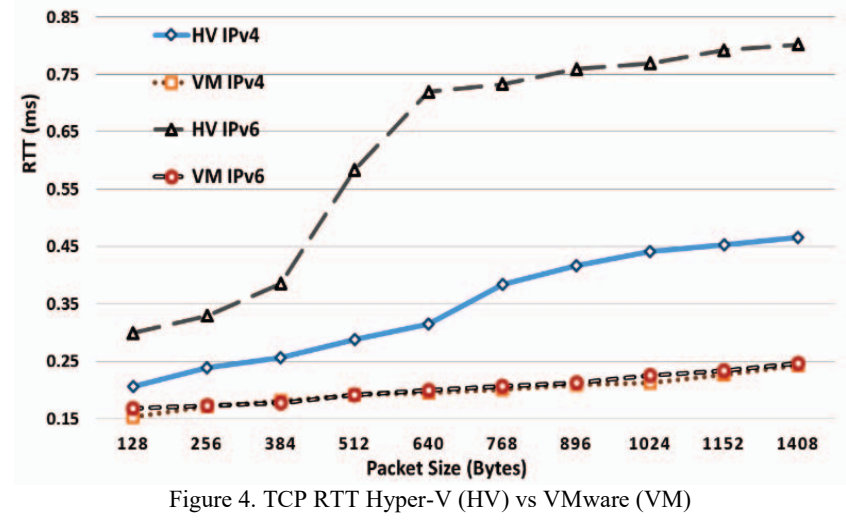

In Figure 4, RTT results were obtained with the same tool used to obtain throughput results for better pinpoint accuracy. Hyper-V (HV) IPV4 RTT results show a slight increase in RTT from packet size 128 to 640 Bytes with a total of $0.5-0.10$ millisecond $(\mathrm{ms})$ increase in RTT. Continuing from packet size 640 to 1408 the RTT results show a gradual incline in RTT with $0.46 \mathrm{~ms}$ recorded in packet size 1408 Bytes, lowest being in packet size 128 at $0.20 \mathrm{~ms}$. VMware (VM) IPv4 results show a minimum increase in RTT from the lowest packet size to the highest packet size. Highest recorded RTT for VMware results are for packet size 1408 Bytes at $0.24 \mathrm{~ms}$ and the lowest one at $0.15 \mathrm{~ms}$ on packet size 128 Byte. We found that Hyper-V TCP IPv4 RTT shows more than half in comparison to VMware TCP IPv4 RTT results showing almost $0.10 \mathrm{~ms}$ difference amongst the two, resulting in VMware IPv4 RTT with the best results. Figure 4 also shows HV TCP IPv6 RTT results show minor increase from packet size 128 to packet size 384 Bytes resulting in total of $0.8 \mathrm{~ms}$ increase in RTT. From packet size 512 to 1408 Bytes, the RTT incline is very high in each packet as the packet size increases. Highest packet size recorded in packet size 1408 Bytes at $0.80 \mathrm{~ms}$ in
HV TCP IPv6 and lowest on packet size 128 at $0.30 \mathrm{~ms}$. VM TCP IPv6 RTT, shows a gradual incline from smallest packet to the largest packet size. Highest RTT recorded for VM TCP IPv6 is $0.25 \mathrm{~ms}$ with the lowest one at $0.17 \mathrm{~ms}$ with an average increase of $0.7 \mathrm{~ms}$ with overall 10 packet sizes. We found that overall in TCP RTT, VM provides less RTT results compared to HV TCP RTT, which is almost as twice as much on every packet size.

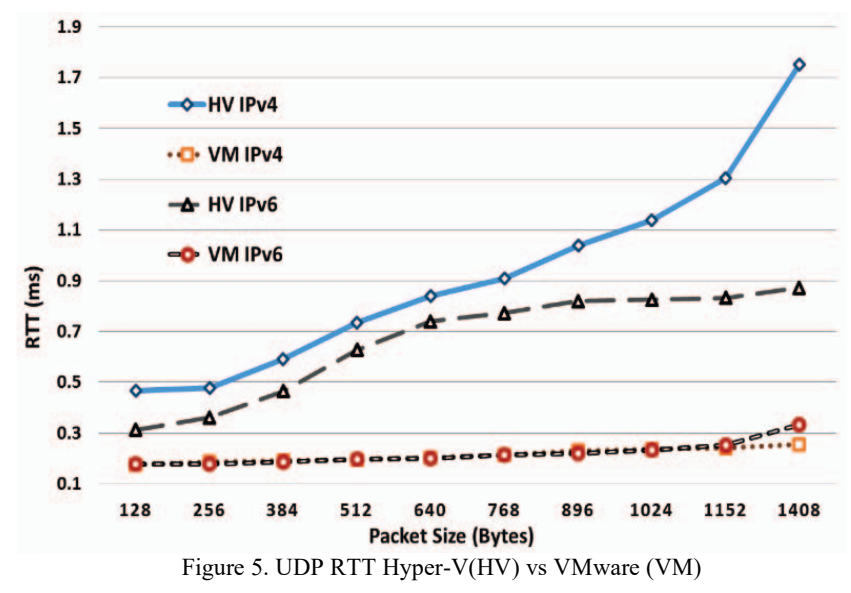

In Figure 5, results for HV UDP IPv4 RTT, the increase is at a high rate from packet size 256 to 1152 Bytes. Averaging a total increase of $0.83 \mathrm{~ms}$ in total. Packet size from 1152 to 1408 Bytes for HV UDP, IPv4 showed a dramatic increase in RTT, with the highest packet size of 1408 Bytes RTT is at $1.75 \mathrm{~ms}$ and at 1152 Bytes measuring at $1.30 \mathrm{~ms}$. Showing an increase of $0.30 \mathrm{~ms}$ between the two packets. Lowest RTT recorded on packet size 128 Bytes at $0.46 \mathrm{~ms}$. We found as the RTT packet size increases the RTT results are higher for each packet size.

For VM UDP-IPv4 RTT results. highest RTT is recorded on packet size 1408 at $0.25 \mathrm{~ms}$ and lowest RTT is recorded on packet size 128 at $0.17 \mathrm{~ms}$. The average increase between lowest to highest packet sizes, is between 0.4-0.8 milliseconds. Overall, we found that VM IPv4 RTT is giving better results than HV UDP-IPv4 RTT results. HV UDPIPv6 RTT results are inclining as the packet size increases. Highest RTT is recorded on packet size 1408 Bytes at 0.87 $\mathrm{ms}$, while a gradual increase in RTT from packet size 128 to 512. Lowest RTT for HV IPv6 is recorded on packet size 128 at $0.31 \mathrm{~ms}$. RTT settling from packet size 640 to 1408 Bytes is not much as the previous packets with an average of $0.10-0.20 \mathrm{~ms}$ increase. VM UDP-IPv6 RTT is quite consistent in rising without any major increases in higher packets. RTT rise from packet size 128 to 1408 Bytes is between $0.10-0.16 \mathrm{~ms}$ in total. Highest recorded RTT for VM UDP-IPv6 is on packet size 1408 Bytes at $0.33 \mathrm{~ms}$ and the lowest RTT is recorded on packet size 1208 at $0.18 \mathrm{~ms}$, it is clear that the results are showing $\mathrm{VM}$ as a better hypervisor in both TCP and UDP using IPv4 and IPv6. 


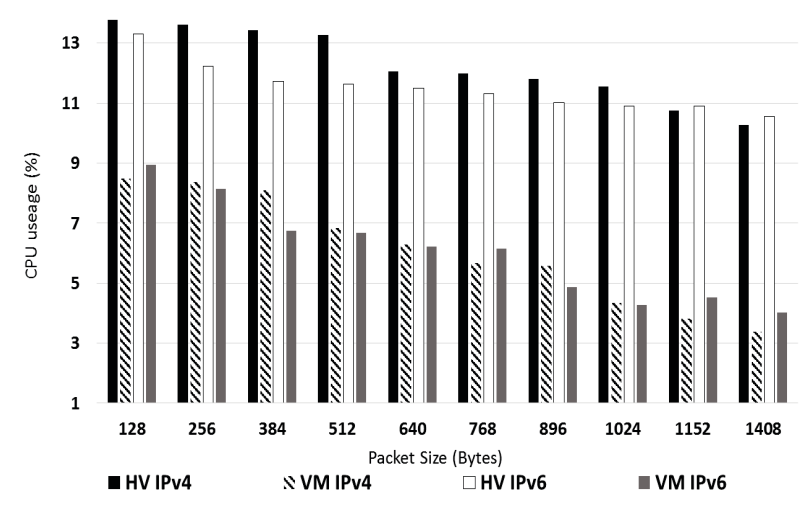

Figure 6 TCP CPU Hyper-V (HV) vs VMware (VM)

Figure 6 shows the results recorded for TCP CPU utilization for Hyper-V and VMware hypervisors. Hyper-V TCP IPv4 CPU utilization is recorded at $13.78 \%$ as the highest for the packet size 128 Bytes. Lowest CPU utilization was recorded on packet size 1408 Bytes at 10.27 \%. Hyper-V TCP IPv4 CPU utilization shows a slight decline on each packet size as the packet size increases. The bigger packets have lower CPU usage as there are less packets involved to process than 128 Byte packets.

VMware TCP IPv4 CPU utilization shows a gradual decline starting from $8.48 \%$ at packet size 128 Bytes and the lowest CPU utilization recorded on packet size 1408 Bytes at $3.39 \%$ showing a reduction of $5.09 \%$ from the smallest packet to the largest packet size in CPU utilization. We found that VMware TCP IPv4 CPU utilization results are showing much lower CPU utilization than Hyper-V TCP IPv4 when measuring throughput. Hyper-V TCP-IPv6 CPU utilization highest recorded figure is on packet size 128 Bytes at $13.30 \%$ CPU utilize and lowest figure recorded on packet size 1408 at $10.54 \%$ utilize. The average amount of decline in CPU utilization for Hyper-V TCP-IPv6 is 2-3\%, from the lowest to the highest packet size. For VMware TCP-IPv6, packet size 128 Bytes has again the highest CPU utilization at $8.9 \%$. We found that even though it is IPv6, the CPU results are much lower than IPv4 for Hyper-V. Lowest recorded CPU utilization for VMware TCP IPv6 is at 4.03\% with an average decline of $3-4 \%$ from packet size 128 to

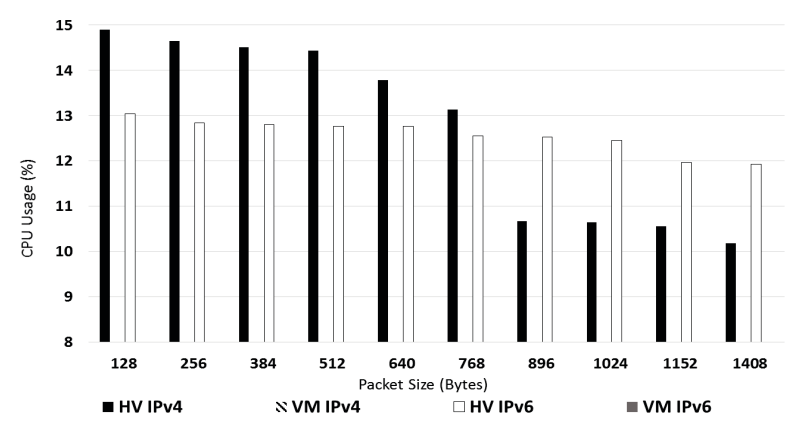

Figure 7. UDP CPU Hyper-V (HV) vs VMware (VM)
1408 Bytes. with overall TCP CPU utilization results. We found that VMware provides the best results in CPU utilization.

Figure 7 shows the results gathered for UDP CPU utilization for Hyper-V. Hyper-V UDP, IPv4 CPU utilizations show a decline from the lowest packet to the highest packet. With $14.90 \%$ recorded on packet size 128 Bytes and lowest CPU utilization recorded on packet size 1408 Bytes at $10.18 \%$ with an average decline between 3$5 \%$. Highest decline was recorded on packet size 768 Bytes at $13.13 \%$ and $10.67 \%$ on packet size 869 Bytes. Hyper-V UDP IPv6 CPU utilization has been on a stable decline throughout the packet sizes, we did not find any impulsive drops in the Utilization. Highest recorded CPU utilization for Hyper-V UDP IPv6 is $13.04 \%$ of packet size 128 Bytes and the lowest recorded on packet size 1408 at $11.93 \%$ with an average of $1-2 \%$ decline overall. As discussed, there are more packets at low packet sizes and therefor it needs more processing resulting in higher CPU usage.

\section{CONCLUSION}

The results that were obtained from these experiments show a great deficiency of difference between results that are mentioned in theory and the results that are obtained practically. With our overall throughput results, it shows us that VMware is able to achieve much higher throughput that Hyper-V (Figure 2). One of the reasons could be Microsoft does not specialize in hypervisors, they have created a Hyper-V as an additional software that Microsoft provides. Whereas as VMware has always been focusing on virtualization from the very beginning, so considerably more development has been implemented into the vSphere hypervisor. Over all RTT results show that Hyper-V is giving higher RTT results than VMWare (Figures 4 and 5). But the delays are in milliseconds and in practice, it should not make noticeable difference. In CPU utilization VMware results are showing that VMware is using very minimal CPU usage comparing it with Hyper-V which is showing very high CPU usage compared to VMware, we found that VMware is able to tolerate the same about of load giving to Hyper-V with much less CPU utilized. Overall, we find that VMware is much more stable and is able to provide higher throughput closest to the theory 1 Gigabit mark.

\section{FUTURE WORKS}

Future works will include doing performance using higher grade hardware to successfully carry out UDP and CPU tests using VMware and Hyper-V. More future works will include doing throughput with 10Gbit Ethernet, evaluating disk management resources and energy consumptions on different hypervisors. 


\section{ACKNOWLEDGEMENT}

The authors would like to thank UNITEC institute of technology for finding the research team and providing the inventory needed.

\section{REFERENCES}

[1] Microsoft, "Virtualization and private cloud," [Online]. Available: http://www.microsoft.com/en-us/server-cloud/solutions/virtualizationprivate-cloud.aspx.

[2] D. Marshall, "Infoworld," 02 November 2011. [Online]. Available: http://www.infoworld.com/article/2621446/servervirtualization/server-virtualization-top-10-benefits-of-servervirtualization.html.

[3] Microsoft, "Hyper-V overview," 31 st March 2015. [Online]. Available: https://technet.microsoft.com/library/hh831531.aspx

[4] VMware, "Vmware," [Online]. Available: https://www.vmware.com/pdf/hypervisor_performance.pdf.

[5] A. Syrewicze, "VMware vs Hyper-V architectural differences," 02 October 2013. [Online]. Available: http://syrewiczeit.com/vmware-vshyper-v-architectural-differences/.

[6] whatmatrix, "Hyper-V vs VMware vSphere," 2015. [Online]. Available:

https://www.whatmatrix.com/comparison/Virtualization.

[7] Torbić, I. Stanković ; B. Đorđević ; V. Timčenko, "Hyper-V and ESXi hypervisors comparison in Windows Server 12 virtual environment", 2018 17th International SymposiumIifotech-Jahorina (INFOTEH), 2018.

[8] V. K. Manik, D. Arora "Performance comparison of commercial VMM: ESXI, XEN, HYPER-V \& KVM”, 3rd International Conference on Computing for Sustainable Global Development, 2016.

[9] H. A. Jabry, L. Liu, Y. Zhu and J. Panneerselvam, "A critical Evaluation of the performance of virtualization technologies," in School of Computing and Mathematics, Derbyshire, 2014.

[10] J. P. Walters, V. Chaudhary, M. Cha, S. Guercio Jr and S. Gallo, "A comparison of Virtualization Technologies for HPC," in Advanced Information Networking and Applications, Buffalo, 2008.

[11] F. Xu, J. Zhou, Y. Wan, and Y. Jiang, "Quantifying performance properties of virtual machine," in Information science and Engineering, Hangzhou, 2008.

[12]Netperf, "Welcome to the Netperf Homepage," http://www.netperf.org/netperf/.

[13] P. Li, S. S Kolahi, M. Safdari, M. Argawe, "Effect of WPA2 security on IEEE $802.11 \mathrm{n}$ bandwith and round trip time in peer-peer wireless local area networks", IEEE Workshops of International Conference on Advanced Information Networking and Applications, 777-782, 2011

[14] B. K Soorty, S. S Kolahi, N. Chand, Q. Zhang "Performance comparison of category 5e vs. category 6 cabling systems for both IPv4 and IPv6 in Gigabit Ethernet", 10th IEEE International Conference on Computer and Information Technology, 1525-1529, 2010 